\title{
Pre-sleep Cognitive Arousal Decreases Following a 4-Week Introductory Mindfulness Course
}

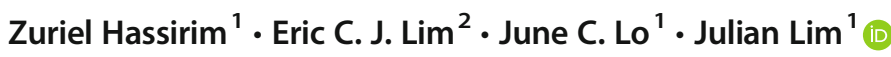

Published online: 20 August 2019

(C) The Author(s) 2019

\begin{abstract}
Objectives Mindfulness-based training has shown potential in reducing anxious and ruminative thoughts before sleep, and improving sleep quality. A majority of experiments on this topic have studied Mindfulness-Based Stress Reduction, or related 8-week programs. In this study, we measured the effects of a 4-week Mindfulness Foundation course on sleep quality assessed via subjective report and actigraphic recording.

Methods Ninety-six participants were recruited from a pool of Mindfulness Foundation course attendees and studied in a waitlist-control design.

Results Although sleep complaints were not an inclusion criterion for this study, sleep quality was poor in the sample as a whole at baseline. We found that sleep quality improved in both groups across the study period $\left(\eta_{\mathrm{p}}{ }^{2}=0.22\right)$, with no statistical difference between groups. In contrast, pre-sleep cognitive arousal was significantly reduced in the treatment, but not the waitlist group $\left(\eta_{\mathrm{p}}{ }^{2}=\right.$ 0.058). Exploratory analysis revealed that reductions in cognitive arousal were correlated with improvements in sleep quality in the treatment group, but not the waitlist group. In the actigraphy data, a small but statistically significant interaction favoring the treatment group was found in the amount of time spent awake during the night (i.e., wake after sleep onset) $\left(\eta_{\mathrm{p}}{ }^{2}=0.070\right)$.

Conclusions Overall, our data demonstrate that some of the benefits to sleep commonly seen over 8 weeks of mindfulness practice can also be observed over a shorter intervention period, and add to the growing body of evidence that mindfulness can help those with poor sleep quality.
\end{abstract}

Keywords Mindfulness $\cdot$ Intervention $\cdot$ Sleep quality $\cdot$ Pre-sleep arousal $\cdot$ Actigraphy $\cdot$ Wake after sleep onset

Poor sleep quality is a common complaint in the general population, even among those who do not meet diagnostic criteria for specific sleep disorders (Yong et al. 2017). Sleeping poorly or insufficiently can lead to acute adverse consequences such as negative mood (Dinges et al. 1997; Haack and Mullington 2005), cognitive impairment (Lim and Dinges 2010; Lowe et al. 2017; Wild et al. 2018), and low quality of life (Strine and Chapman

Electronic supplementary material The online version of this article (https://doi.org/10.1007/s12671-019-01217-4) contains supplementary material, which is available to authorized users.

Julian Lim

julian.lim@duke-nus.edu.sg

1 Center For Cognitive Neuroscience, Neuroscience and Behavioral Disorders Department, Duke-NUS Medical School, 8 College Road, Singapore 169857, Singapore

2 Brahm Centre, Ren Ci Hospital, Singapore 329562, Singapore
2005). More importantly, chronic poor sleepers are at higher risk of developing more serious health problems such as diabetes (Cappuccio et al. 2010a) and cardiovascular disorders (Cappuccio et al. 2011), and have a higher overall mortality risk (Cappuccio et al. 2010b).

While treatments such as cognitive behavioral therapy for insomnia (CBT-I) (Morin 1993; Schutte-Rodin et al. 2008) are recommended for those diagnosed with primary sleep disorders, these options may not be appropriate for subclinical cases due to their limited availability and scalability. Instead, mindfulness-based programs may be more suitable for those with sub-threshold levels of sleep disturbance, or for healthy sleepers aiming to improve their sleep quality further.

Mindfulness training enhances metacognitive processes (higher-order functions that monitor and assess cognition) (Kok and Singer 2017; Teasdale 1999), which raise the awareness of the contents of conscious experience (Jankowski and Holas 2014). This enhanced 
processing may in turn improve sleep quality by promoting a non-automatic "adaptive stance" to arousing or maladaptive sleep-related cognitions (Ong et al. 2012), and reducing processes such as rumination or avoidance. For example, mindful individuals may be more proficient at adopting a neutral, non-reactive attitude towards negative thoughts about sleep, leading to a calmer and less anxious response to such thoughts.

In line with this theory, trait mindfulness has been associated with sleep quality in healthy individuals (Howell et al. 2008) and mindfulness training has been shown to improve sleep outcomes in patients with primary insomnia (Gross et al. 2011; Ong et al. 2014; Zhang et al. 2015) and depression (Britton et al. 2010). Mindfulness training may act on sleep quality via reducing the level of arousal prior to sleep. High levels of pre-sleep cognitive arousal are associated with longer sleep onset latency, or the time taken to initiate sleep before getting into bed (Tang and Harvey 2004), and lower levels of arousal may help to reduce nighttime awakenings, and promote longer continuous episodes of nocturnal sleep (Chen et al. 2011). Recent studies (Greeson et al. 2018; Ong et al. 2014) have shown that reduction in cognitive arousal prior to sleep correlates with improvements in sleep quality over 8week mindfulness-based interventions. Similarly, Blake et al. (2017) reported that pre-sleep cognitive arousal mediated improvements in sleep quality in a study of mindfulness-based training for at-risk adolescents. In sum, there is preliminary evidence to suggest that 8week interventions may have effects on improving sleep by way of decreasing pre-sleep arousal.

Shorter mindfulness programs may have wider reach and greater acceptability than standard 8-week courses; in support of this, a 4-week mindfulness course offered by the Brahm Centre in Singapore has almost twice the enrolment of the traditional 8-week mindfulness-based stress reduction (MBSR) run at that Centre (E.C.J. Lim, personal communication, February 27, 2018). Furthermore, 8-week studies have relatively high attrition rates (Chang et al. 2004; Williams et al. 2001), suggesting that shorter courses may be more suitable for novices to meditation who do not have urgent complaints.

This study aimed to investigate the effects of a 4week introductory mindfulness intervention on subjective and objective sleep quality in a community sample. The primary hypothesis of the study was that the treatment group would show improvements in both subjective and objective (actigraphic) sleep quality compared with the waitlist group, and that this improvement would be mediated by decreased cognitive arousal prior to sleep.

\section{Methods}

\section{Participants}

Enrollees in the Mindfulness Foundation Course (see below) were invited to participate in the study if they were between 15 and 75 years of age, with no history of sleep disorders, substance abuse, or long-term use of sedative meditation. Subjects were excluded if they were currently engaged in shift work, or if they were planning international travel within 2 weeks prior to any study session.

Our a priori target sample size for this study was 88 , based on an estimated small-medium effect size (Cohen's $f=0.15 /$ eta-squared $=.022$ ) and power of $\beta=0.8$. Figure 1 depicts the CONSORT diagrams of participant flow through the study. A total of 127 people were contacted, out of which 57 were eventually assigned to the treatment group, and 39 to the waitlist group. Four participants dropped out of the experiment prior to study completion, and 1 participant's data was removed because they started attending a separate Mindfulness-Based Stress Reduction class during the waitlist period.

This study was approved by the National University of Singapore Institutional Review Board, and all participants provided written informed consent. Volunteers below 18 years of age additionally obtained parental consent to participate in this research. Participants were compensated Singapore $\$ 50$ 60 for taking part in the study (or a pro-rated amount for noncompleters).

Group Assignment Participants were allocated to groups in a blocked fashion (Efird 2011) based on course enrolment, with each block consisting of two rounds of the Mindfulness Foundation Course. For the first 3 blocks, participants ( $N=$ 39) were randomized to the intervention or waitlist group. Those in the intervention group were assigned to attend the first course in the block, and those in the waitlist group were assigned to the second. Due to a change in logistical arrangements with the course providers, we were no longer able to carry out random group assignment after the first 3 blocks. The remaining 56 participants were thus free to select the course within each block they wished to enroll in, though at the time of selection they were not aware of which research group they would be placed into. For these latter blocks, participants who selected the first course in each block formed the intervention group.

\section{Procedure}

Figure 2 shows a schematic of the study schedule. Participants in the intervention group attended two in-laboratory sessions that were held approximately 1 week before and after the Mindfulness Foundation Course. During these sessions, they 
a

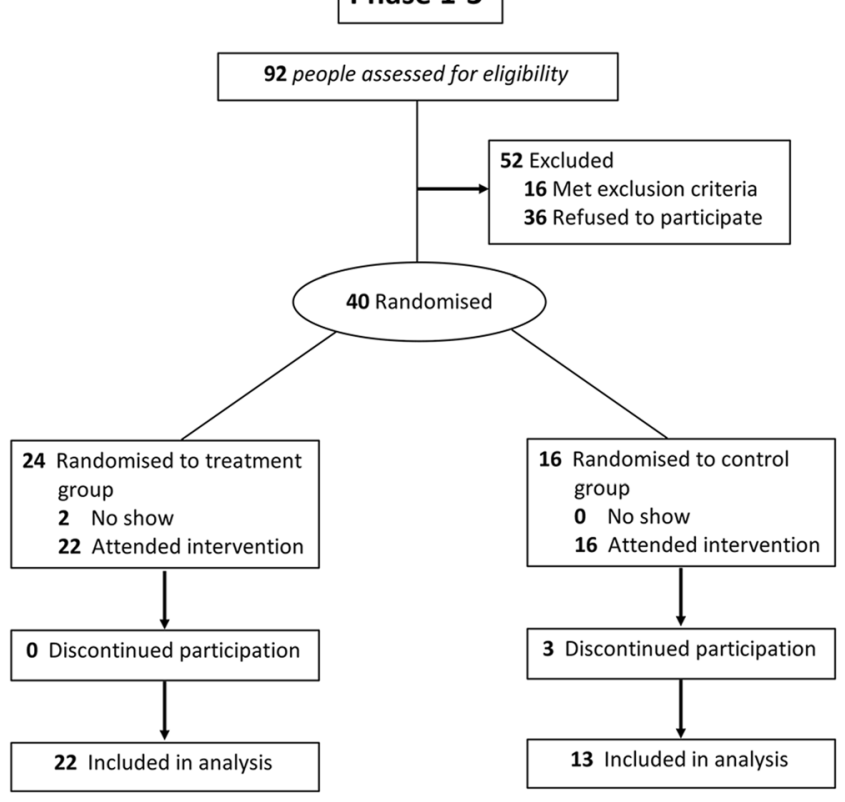

b

\section{Phase 4-6}

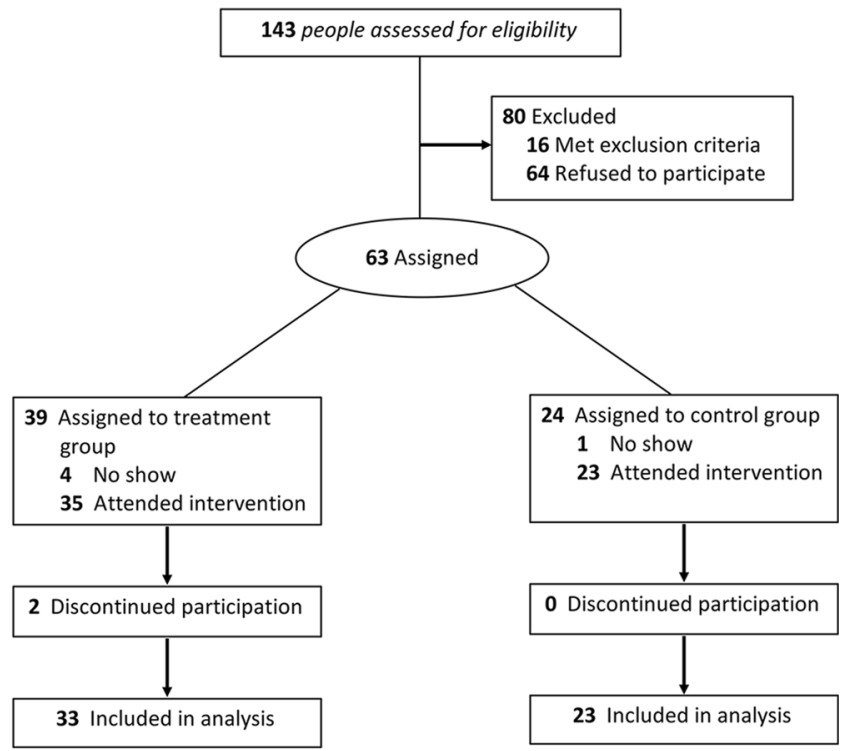

Fig. 1 CONSORT diagram depicting the flow of study participants. a Participants in blocks 1-3 $(N=39)$ were randomized to condition, and $\mathbf{b}$ the remaining participants were assigned to group based on whether their selected course was the first or the second within a block

completed all questionnaires and performed a 10 -min sustained attention test (results not reported here).
Actigraphy data were collected during the weeks between the testing session and the first/last mindfulness class.

\section{Treatment Group}
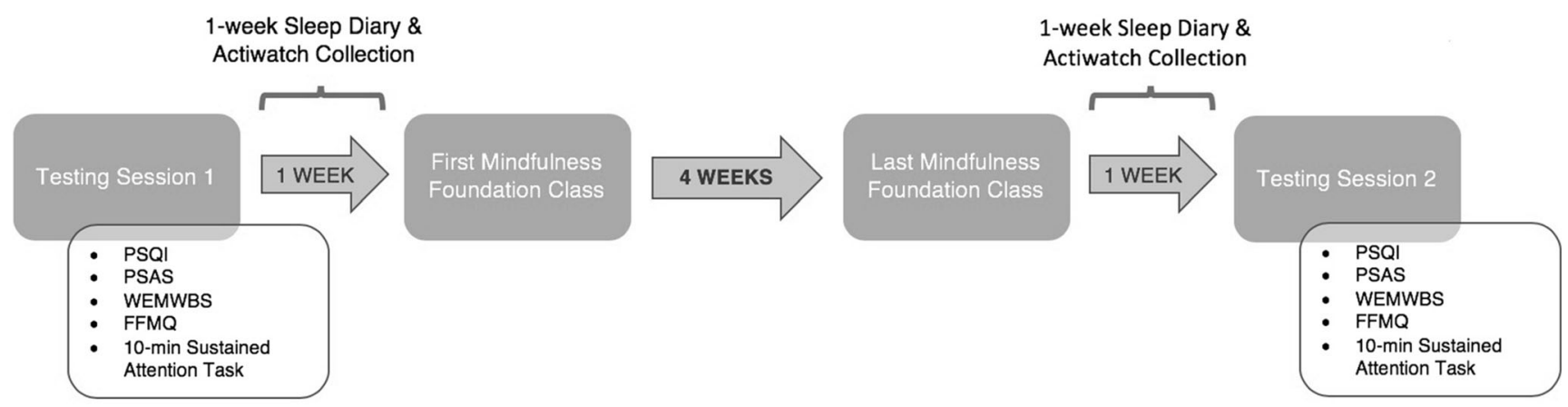

\section{Waitlist Control Group}
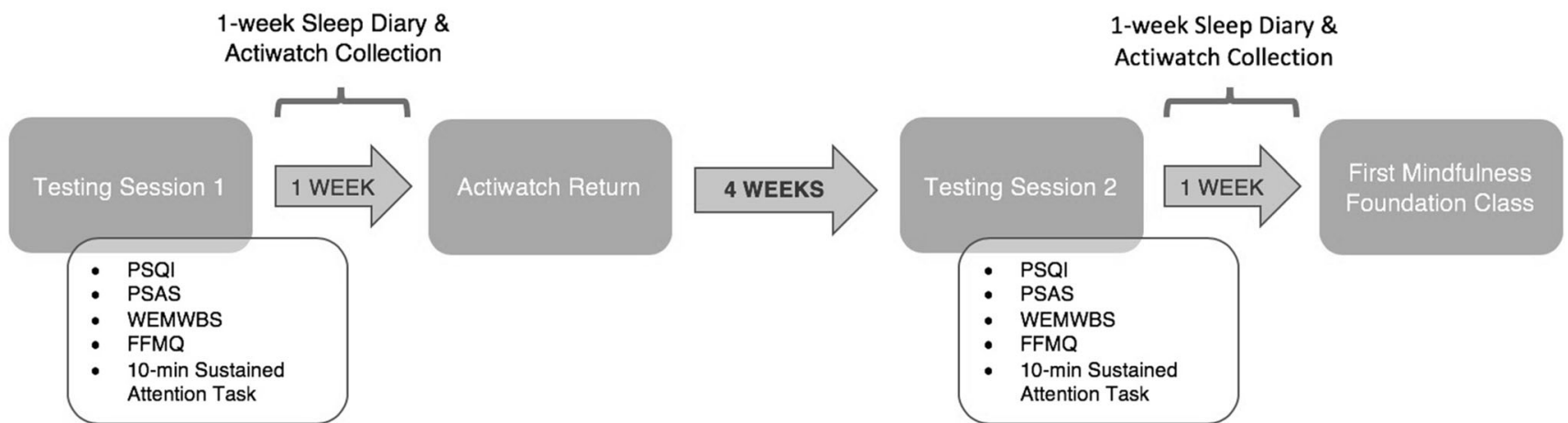

Fig. 2 Study schedule. PSQI, Pittsburgh Sleep Quality Inventory; PSAS, Pre-Sleep Arousal Scale, WEMWBS, Warwick-Edinburgh Mental Wellbeing Scale; FFMQ, Five-Facet Mindfulness Questionnaire 
Participants in the waitlist group underwent a similar data collection schedule without attending the mindfulness course between the two laboratory sessions. Note that the interval between the weeks of actigraphic measurement was identical between the groups ( 5 weeks), but that the intervals between questionnaire completion differed by a week (6-week interval for the intervention group; 5-week interval for the waitlist group). Data collection was performed in Duke-NUS Medical School, and the Mindfulness Foundation Course was conducted in the Brahm Centre at Ren Ci Hospital, Singapore. Data were collected between February 2017 and April 2018.

Intervention The Mindfulness Foundation Course is a weekly 2-hour, 4-session group-based mindfulness intervention designed by the Brahm Centre. Each session consisted of didactic teaching, group discussion, inquiry, and formal mindfulness practices. Participants engaged in $10 \mathrm{~min}$ of formal mindfulness practices including body scan, breath awareness, loving-kindness meditation, and informal mindfulness practices of mindful eating and mindfulness of daily activities both in session and on a daily basis. Participants were provided with weekly session handouts and guided audio meditation tracks for their daily practice (10-15 min). The mindfulness curriculum was taught by an experienced mindfulness teacher who holds a qualification from the Centre For Mindfulness (CFM), University of Massachusetts Medical School, and has trained over 3000 people.

The themes for the 4 sessions were "Introduction to Mindfulness," "Be Right Where You Are," "Our Storytelling Minds," and "Cultivating Kindness." In the first session, participants were introduced to mindfulness and explored some of the foundational attitudes of mindfulness practice. In the second session, participants explored the habits of the mind, identified their stress triggers and learn the value of being present with awareness. The third session invited participants to investigate the power of their storytelling minds and how it may have affected their stress levels. The final session ended with an invitation to work with difficult emotions and thoughts through cultivation of kindness towards oneself and others. There was no explicit focus on sleep education or improving sleep quality in the course.

\section{Measures}

Pittsburgh Sleep Quality Index The primary subjective outcome measure for this study was the Pittsburgh Sleep Quality Index (PSQI), a scale that assesses sleep quality based on self-reported problems and difficulties (Buysse et al. 1989). A PSQI global score of more than 5 is indicative of poor sleep.

Pre-Sleep Arousal Scale The Pre-Sleep Arousal Scale (PSAS) is a secondary measure in this study (Nicassio et al. 1985). The
PSAS measures somatic and cognitive arousal in the time period that is right before sleep. The measure demonstrated good internal consistency for both the cognitive and somatic subscales in populations of normal sleepers (cognitive $\alpha=$ 0.67 and somatic $\alpha=0.84$ ) and insomniacs (cognitive $\alpha=$ 0.76 and somatic $\alpha=0.81$ ). The PSAS was only administered from study block 2 onwards (i.e., data were not available from the first 13 participants). In the current study, internal consistency was good at $\alpha=0.72$ for both subscales.

Warwick-Edinburgh Mental Wellbeing Scale The WEMWBS is a 14-item scale which has been developed to monitor general mental wellbeing and has been used in the evaluation of programs aiming to improve mental wellbeing (Tennant et al. 2007). Respondents indicate how well they fell mentally on a Likert scale from 1 to 5 . The WEMWBS has high internal consistency $(\alpha=0.91)$. Excellent internal consistency was also observed in the current sample: $\alpha=0.92$.

Five Facet Mindfulness Questionnaire The FFMQ is a 39-item measure consisting of five subscales used to assess the construct of mindfulness (Baer et al. 2008). The five component skills that are thought to constitute mindfulness are observing, describing, acting with awareness, non-judging of inner experience, and non-reactivity to inner experience. The five scales of the FFMQ have adequate to good internal consistency, with alpha coefficients ranging from 0.75 to 0.91 . In the current study, internal consistency was high for the total FFMQ score: $\alpha=0.90$.

Morningness-Eveningness Questionnaire The MEQ is a 19item scale to measure which time period in a day a person's circadian rhythm is at peak alertness (Horne and Ostberg 1976). Questions are framed in a preferential manner, where the respondent is asked to indicate when, for example, he/she would prefer to wake up or start sleep, rather than when he/she actually does.

Demographic information Demographic information was also collected from each participant (age, gender, occupation, race, marital status, education level, household income).

Actigraphy Actigraphy (Actiwatch 2, Philips Respironics Inc., Pittsburgh, PA), which estimates sleep and wake cycles by monitoring movement and light exposure, was measured for 1 week prior to and directly following the intervention period. These data were analyzed if the participant was able to provide at least 3 weekdays and 1 weekend of scorable records in each data collection period.

Sleep and wake times on the actigraphic data were marked by two experienced research assistants. We standardized this scoring via a protocol that prioritized actigraphy markers, then sleep diaries, and finally a distinct drop in movement and light levels. Discrepancies of $>1 \mathrm{~h}$ between scorers on any night 
were arbitrated by one of the senior investigators. Scorers were blind to the group assignment of participants. Our primary outcome variables of interest derived from the actigraphy were sleep onset latency (SOL), wake after sleep onset (WASO), and sleep efficiency (SE). These variables were calculated automatically using Actiware software (version 6.0.2). For completeness, we also calculated and report time in bed (TIB) and total sleep time (TST). Variables were weighted by weekdays/weekends so that this ratio was $5: 2$ for all participants, regardless of how many nights of scorable actigraphy data they contributed.

Six actigraphy datasets were discarded prior to analysis because of equipment malfunctions or insufficient nights of good quality data. Three further participants' data were removed as outliers in either WASO or SOL ( $>3$ SD from the mean). Thus, 87 datasets (treatment $=53$, waitlist $=34$ ) entered the final analysis.

Participants were asked to maintain sleep diaries during the week of actigraphic recording. On these, they recorded daily sleep and wake times, subjective sleep latency, number and duration of nighttime awakenings, and daytime naps (if any). Sleep diary data were used to inform the actigraphic scoring and are not analyzed here as outcome variables.

\section{Data Analyses}

Statistical analysis was performed in IBM SPSS 24.0 for Mac. Baseline participant characteristics were compared using independent samples $t$ tests. We conducted intent-to-treat (ITT) analysis by filling in missing data with the pooled average of five multiple imputations. Outliers ( $>3 \mathrm{SD}$ from the mean) were removed prior to inferential analysis. To test the effect of the intervention, outcome variables were subjected to repeated-measures ANCOVA with group as a betweensubject variable, and age, gender, and years of education as covariates. We considered an interaction at the level of $p<.05$ to be a statistically significant benefit of treatment. Post hoc analyses comparing pre- and post-intervention scores in both groups were carried out if we found such a benefit, or a main effect of time. Results from the ITT analysis were comparable with the completer analysis, which is reported in the Supplemental Material.

\section{Results}

\section{Baseline Characteristics}

Table 1 contains the baseline characteristics of participants in the treatment and waitlist groups. Groups did not differ in mean age, gender, racial balance, and morningnesseveningness preference. However, participants in the treatment group had significantly more years of education (mean
Table 1 Baseline characteristics

\begin{tabular}{|c|c|c|c|c|c|}
\hline \multirow[t]{2}{*}{ Variable } & \multicolumn{2}{|c|}{ Treatment } & \multicolumn{2}{|l|}{ Waitlist } & \multirow[t]{2}{*}{$p$} \\
\hline & Mean & $\mathrm{SD}$ & Mean & $\mathrm{SD}$ & \\
\hline$N$ & 57 & - & 39 & - & \\
\hline \multicolumn{6}{|l|}{ Demographics } \\
\hline Age & 47.7 & 13.8 & 51.8 & 16.0 & .11 \\
\hline Gender (male) & 15 & - & 14 & - & .30 \\
\hline Education (years) & 14.6 & 2.8 & 13.4 & 2.3 & $.03 *$ \\
\hline \multicolumn{6}{|l|}{ Race } \\
\hline Chinese & 54 & - & 35 & - & \\
\hline Indian & 1 & - & 2 & - & \\
\hline Others & 1 & - & 1 & - & \\
\hline Not reported & 1 & - & 1 & - & \\
\hline \multicolumn{6}{|l|}{ Questionnaires } \\
\hline MEQ & 52.56 & 8.53 & 51.79 & 9.64 & .69 \\
\hline PSQI & 6.93 & 3.25 & 7.15 & 3.55 & .75 \\
\hline PSAS (somatic) & 10.39 & 2.69 & 10.30 & 2.79 & .88 \\
\hline PSAS (cognitive) & 15.17 & 4.97 & 15.93 & 4.61 & .50 \\
\hline WEMWBS & 50.82 & 8.10 & 51.44 & 7.55 & .71 \\
\hline \multicolumn{6}{|l|}{ FFMQ } \\
\hline Observing & 25.48 & 4.73 & 27.51 & 4.53 & $.04 *$ \\
\hline Describing & 26.39 & 5.71 & 28.36 & 5.04 & .09 \\
\hline Acting with awareness & 26.38 & 6.17 & 27.28 & 6.27 & .48 \\
\hline Non-judging & 24.27 & 4.89 & 25.38 & 5.21 & .29 \\
\hline Non-reactivity & 21.45 & 4.22 & 22.46 & 4.67 & .27 \\
\hline \multicolumn{6}{|l|}{ Sleep diaries } \\
\hline TIB (min) & 459.89 & 56.00 & 435.10 & 48.06 & $.05 *$ \\
\hline TST (min) & 432.45 & 47.37 & 400.84 & 57.18 & $.01^{*}$ \\
\hline SOL (min) & 16.44 & 12.23 & 22.14 & 28.40 & .62 \\
\hline WASO (min) & 11.00 & 12.94 & 12.12 & 16.34 & .73 \\
\hline $\mathrm{SE}(\%)$ & 94.22 & 0.55 & 92.22 & 9.19 & .19 \\
\hline \multicolumn{6}{|l|}{ Actigraphy } \\
\hline TIB (min) & 457.13 & 53.20 & 433.61 & 56.60 & $.05 *$ \\
\hline TST (min) & 375.10 & 54.21 & 350.29 & 57.00 & $.03 *$ \\
\hline SOL (min) & 10.18 & 9.15 & 11.05 & 9.24 & .67 \\
\hline WASO (min) & 59.57 & 19.95 & 59.58 & 22.27 & .72 \\
\hline SE $(\%)$ & 81.10 & 6.82 & 80.77 & 6.51 & .82 \\
\hline
\end{tabular}

Chn Chinese, Ind Indian, Mal Malay, Oth others, MEQ MorningnessEveningness Questionnaire, $P S Q I$ Pittsburgh Sleep Quality Index, PSAS Pre-Sleep Arousal Scale, WEBWMS Warwick-Edinburgh Mental Wellbeing Scale, FFMQ Five Facet Mindfulness Questionnaire, TIB time in bed, TST total sleep time, $S O L$ sleep onset latency, WASO wake after sleep onset, $S E$ sleep efficiency. * $p<.05$

$(\mathrm{sd})=14.6(2.8)$ vs. $13.4(2.3)$ years $)$ than the waitlist group. Because of this, we included education as a covariate in our subsequent ANCOVA analysis. The treatment group also had lower scores on the observing subscale of the FFMQ, and significantly more time in bed and total sleep time at baseline. Importantly, the groups did not differ on any of the primary outcome variables. 


\section{Class Attendance}

Attendance to the program was good, with a mean (sd) of 3.73 (0.58) sessions attended overall. We planned to exclude participants from analysis if they attended fewer than 2 of the 4 class sessions; in practice, all but one of the completers attended at least 3 sessions, with the remaining completer attending 2 out of 4 .

\section{Five Facet Mindfulness Questionnaire}

The FFMQ was used as a manipulation check to assess whether the intervention improved self-reported mindfulness. One participant did not complete this questionnaire, and one outlier was removed prior to analysis. Supplementary Figure 1 depicts the FFMQ data separated into its five subscales. In summary, we observed a significant treatment by time interaction in three of the five subscales of small-to-medium effect size (acting with awareness: $F_{1,90}=4.11, p=.046, \eta_{\mathrm{p}}{ }^{2}=.044$; non-judging: $F_{1,90}=8.82, p=.005, \eta_{\mathrm{p}}{ }^{2}=.089$; non-reactivity: $F_{1,90}=4.88$, $\left.p=.04, \eta_{\mathrm{p}}{ }^{2}=.051\right)$, with directionally similar but nonsignificant results on the other two (observing: $F_{1,90}=3.27$, $p=.07$; describing: $F_{1,90}=2.54, p=.11$ ). Post hoc analyses showed that the changes were driven by increases in the treatment but not the waitlist group (acting with awareness: $t_{56}=-$ $4.53, p<.001$; non-judging: $t_{56}=-3.63, p<.001$; non-reactivity: $\left.t_{56}=-3.92, p<.001\right)$.

\section{Pittsburgh Sleep Quality Inventory}

Our primary outcome measure in the self-report data was global PSQI scores. Scores greater than 5 on this scale indicate poor-quality sleep (Buysse et al. 1989), and participants in both groups reported poor sleep on average at baseline, although we did not select the sample based on this criterion. We observed a significant main effect of time driven by improvements in sleep quality in both groups over the study period $\left(F_{1,91}=25.21, p<.001, \eta_{p}{ }^{2}=0.22\right)$. However, there was no significant interaction between improvements in the treatment and waitlist groups $\left(F_{1,91}=1.05, p=.31\right)$ (Fig. 3). Post hoc tests confirmed that significant reductions in PSQI were seen in both experimental groups (treatment: $t_{56}=4.25$, $p<.001$; waitlist: $t_{38}=3.27, p=.002$ ).

\section{Pre-Sleep Arousal Scale}

We measured self-reported somatic and cognitive arousal prior to sleep using the PSAS. In the ANCOVA analysis, we found a significant interaction in cognitive arousal with a small-medium effect size $\left(F_{1,90}=4.71, p=.03\right.$, $\left.\eta_{\mathrm{p}}{ }^{2}=0.058\right)$ (Fig. 4), but no interaction in somatic arousal $\left(F_{1,90}=3.49, p=.06\right)$. Post hoc analysis of pre-sleep cognitive arousal showed a significant reduction for the

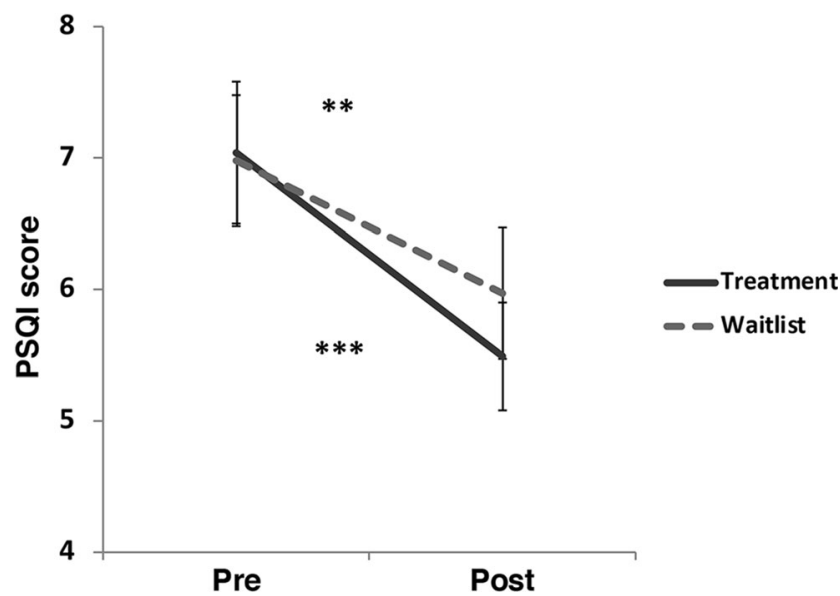

Fig. 3 Subjective sleep quality improved significantly over time in both groups. Scores are estimated marginal means of repeated-measures ANCOVA, and bars are standard errors. $* * p<.01$, *** $p<.001$ (asterisks denote significant differences in post hoc $t$ tests)

treatment but not the waitlist group (treatment: $t_{50}=3.17$, $p=.001$; waitlist: $t_{30}=0.20, p=.84$ ).

Since there was no effect of treatment group on change in PSQI, we could not conduct mediation analysis as planned. Instead, we used Pearson's correlations to test for associations between the change in sleep quality and change in pre-sleep arousal over time. We found a significant correlation between change in pre-sleep cognitive arousal and sleep quality in the treatment group $(r=.37, p=.007)$ but not the waitlist group $(r=-.05, p=.81)$ (Supplementary Figure 2). The difference between the strengths of these correlations differed significantly ( $z=1.81, p=.04$, one-tailed). There was no association between change in pre-sleep somatic arousal and change in sleep quality in either group (treatment: $r=.13, p=.38$; waitlist: $r=.19, p=.31$ ).

\section{Warwick-Edinburgh Mental Wellbeing Scale}

Mental well-being was assessed using the WEMWBS. We observed greater increases in well-being in the treatment group compared with the control group, but this interaction did not reach statistical significance $\left(F_{1,91}=1.79, p=.19\right)$.

\section{Actigraphy}

Our three primary variables of interest in the actigraphy were sleep onset latency (SOL), wake after sleep onset (WASO), and sleep efficiency (SE) (Fig. 5). No significant changes were observed in $\operatorname{SOL}\left(F_{1,82}=0.03, p=.87\right)$ and SE $\left(F_{1,82}=0.39, p=.54\right)$. We observed a significant interaction driven by a numerical decrease in WASO in the treatment group and a numerical increase in the waitlist group $\left(F_{1,82}=6.18, p=0.015, \eta_{\mathrm{p}}{ }^{2}=0.070\right)$. However, post hoc tests showed that neither of the main effects was statistically 
Somatic subscale

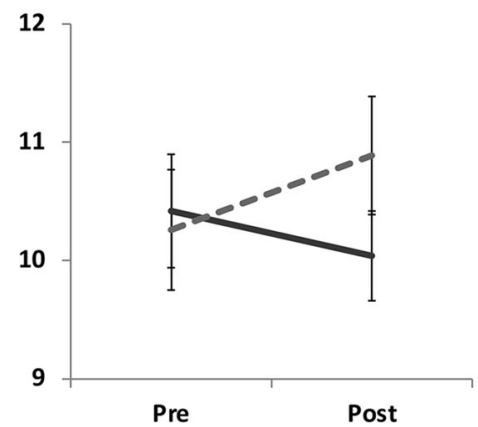

Fig. 4 Pre-sleep cognitive arousal improved significantly over time in the treatment group but not the waitlist group. In contrast, no interaction effect was observed for pre-sleep somatic arousal. Scores are estimated marginal

significant on their own (treatment: $t_{33}=1.43, p=.16$ waitlist: $t_{33}=-1.51, p=.14$ ).

In additional analysis, we interrogated time in bed (TIB) and total sleep time (TST). We found a significant interaction in both of these variables (TIB: $F_{1,82}=9.69, p=0.003, \eta_{\mathrm{p}}{ }^{2}=$ 0.106; TST: $\left.F_{1,82}=6.11, p=0.015, \eta_{\mathrm{p}}{ }^{2}=0.069\right)$ which was driven by decreasing TIB/TST in the treatment group and increasing TIB/TST in the waitlist group.

\section{Discussion}

The study tested the hypothesis that a 4-week general mindfulness intervention would improve sleep quality in a community sample. In brief, we found that subjective sleep quality improved
Cognitive subscale

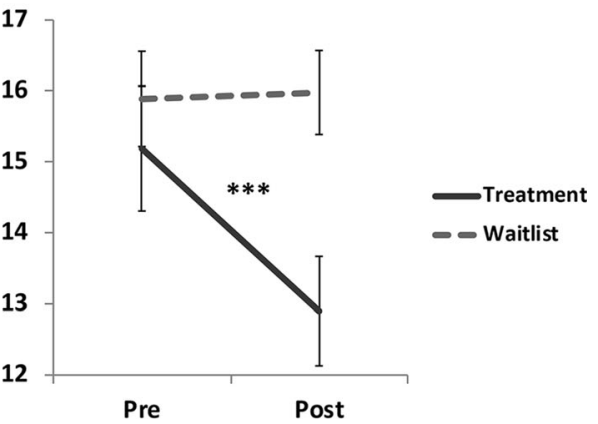

means of repeated-measures ANCOVA, and bars are standard errors. $*^{* * *} p<.001$ (asterisks denote significant differences in post hoc $t$ tests)

across both the treatment and waitlist groups. Pre-sleep cognitive arousal decreased significantly for the treatment but not the waitlist group. Finally, a statistically significant interaction favoring the treatment group was observed for actigraphically measured WASO. We discuss each of these results in turn.

\section{Self-reported Mindfulness Increased in the Treatment Group Only}

Analysis of the FFMQ demonstrated that the 4-week intervention was effective in increasing self-reported mindfulness, with significant effects of treatment in 3 of 5 FFMQ subscales: acting with awareness, non-judging, and non-reactivity. The cultivation of foundational attitudes, in particular non-judging and acceptance, was a key emphasis in the mindfulness-based
Fig. 5 Actigraphic measurement revealed a significant time by group interaction in wake after sleep onset, and no differences in sleep onset latency or sleep efficiency
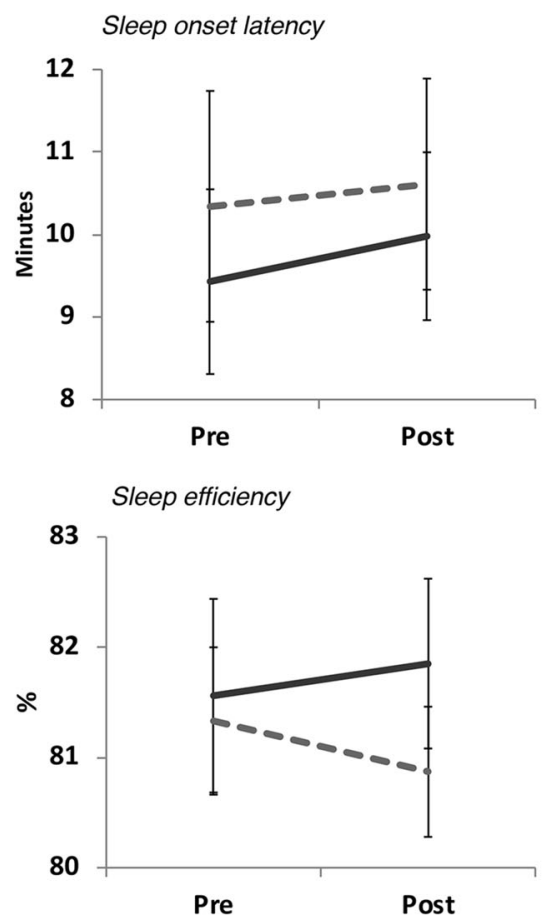
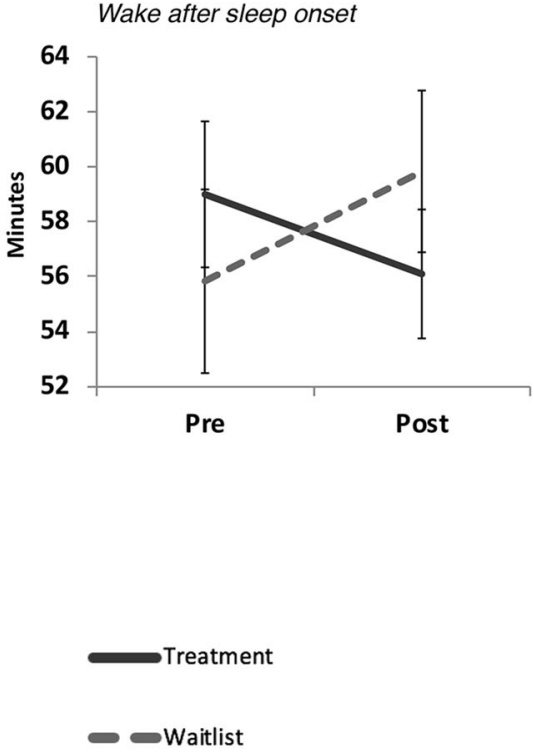
intervention, and participants were invited to bring awareness to moments where these could be practiced and applied in their daily lives. This may account for the relatively larger changes in these three particular FFMQ subscales.

\section{Subjective Sleep Quality Improved in Both Experimental Groups}

PSQI scores indicated that participants reported poor/insufficient sleep on average at baseline, despite the fact that this was not an inclusion criterion for study enrolment. This observation is line with survey data showing that developed Asian nations such as Japan, South Korea, and Singapore have the shortest sleep times internationally, at just under 7.5 hours a night (Walch et al. 2016). Separate local studies have found that $44 \%$ of adult visitors to local clinics have insufficient sleep on weekdays (Tan et al. 2016), and that the prevalence of poor sleep (PSQI > 5) in Singapore is approximately 1 in 4 (Tan et al. 2017). These figures notwithstanding, a higher-than-expected $63 / 96$ of our participants reported PSQI scores greater than 5 at baseline, suggesting that poor sleepers were self-selecting into either the mindfulness program or our research study.

Repeated-measures ANCOVA revealed significant decreases in PSQI scores across both the treatment and waitlist groups. This pattern of results suggests that improvements in the treatment group were in part due to non-experimental factors such as spontaneous remission, maturational influences, or the observer effect. Participating in sleep research may also have triggered self-monitoring behavior and reactivity (Kazdin 1974) in both groups, which could account for the improvements seen in the waitlist participants (Todd and Mullan 2014). In the context of prior studies, the null finding in this experiment suggests that improving subjective sleep quality in a meaningful way may require longer (Black et al. 2015; Britton et al. 2010; Greeson et al. 2018) or more targeted interventions (Ong et al. 2014).

\section{Pre-sleep Cognitive Arousal Improved and Was Associated with PSQI Improvements in the Treatment Group}

In contrast to the PSQI, we found significant reductions in presleep cognitive arousal for the treatment group but not the waitlist group. Interestingly, we also found that reductions in cognitive arousal were correlated with PSQI reduction in the treatment group alone. These results support our hypothesis that mindfulness acts on improving sleep by mitigating maladaptive emotion regulation processes and arousal-producing cognitions (Ong et al. 2012).

Cognitive and somatic hyperarousal are key features of insomnia that maintain and perpetuate the cycle of poor sleep and daytime impairment (Bonnet and Arand 1997; Harvey 2002). Mindfulness techniques aim to reduce this arousal by strengthening metacognition, which can help individuals change their relationship with their dysfunctional thoughts about sleep without necessarily trying to understand or challenge their content (Ong et al. 2012). For instance, developing a non-attached and non-reactive stance to sleep-related cognitions may reduce the anxiety they would otherwise elicit.

Several studies to date have shown a relationship between mindfulness training and reductions in cognitive arousal. Ong et al. (2014) reported reduced PSAS scores with MBSR and MBT-I compared with a self-monitoring control. In the SENSE study, Blake et al. (2017) showed that both somatic and cognitive arousal (measured by the PSAS) were mediators of change in four key outcome variables (objective SOL, subjective SE, PSQI global score, and anxiety). A recent study (Greeson et al. 2018) showed that unwanted intrusive thoughts and thought suppression, both constructs related to cognitive arousal, each accounted for a significant portion of the negative correlation between change in mindfulness and change in sleep disturbance following an 8-week mindfulness intervention. Our own findings are well in line with these results.

Our data support a model in which PSQI reductions were driven by decreases in cognitive arousal in the treatment group only. However, as this is only a correlational and not a mediation analysis, we cannot make strong claims about causation. Our findings also imply that significant reductions in pre-sleep arousal are observable earlier than and may be a precursor to meaningful improvements in sleep quality. Future longitudinal research with sampling at multiple time points could be used to test the causative relationships between these two variables.

\section{Mindfulness Training Reduced Actigraphically Measured WASO}

Objective measures of sleep often do not correlate well with subjective measures. For example, Grandner et al. (2006) found no significant correlation between PSQI global scores and actigraphic measures in either younger or older adults over a period of a week. Landry et al. (2015) also showed a lack of agreement between subjective measures and actigraphy over 14 days in older adults. These data indicate that subjective and objective measures of sleep capture unique aspects of information regarding sleep quality, and motivate the investigation of these two types of data separately.

Previous studies measuring actigraphic changes with mindfulness training have yielded mixed results. In the SENSE study, 123 adolescents were randomized into either a sleep improvement intervention with elements of mindfulness training or a "study skills" program. Adolescents in the Sleep SENSE arm improved significantly more on actigraphic SOL, but not SE, WASO, or TST (Blake et al. 2017). Another RCT of mindfulness-based treatment for insomnia (MBT-I) on patients with primary insomnia also found significant benefit for actigraphic TST and decreases in total wake time (Ong et al. 2014). In contrast, Lengacher et al. (2015) reported only trend- 
level $(p>.07)$ changes in SOL and WASO following a 6-week mindfulness-based stress reduction (MBSR) program compared with treatment-as-usual. Finally, in a smaller trial on urban youth, Sibinga et al. (2013) reported no differences between the MBSR and control (health education) groups on any actigraphic variables. In sum, a clear picture has yet to emerge on whether mindfulness training affects objectively measured sleep, and which variables are the most sensitive to this change.

In the current study, we observed a significant group by time interaction in WASO, with a small increase $(\sim 4 \mathrm{~min})$ in the waitlist group, and a small decrease $(\sim 3 \mathrm{~min})$ in the treatment group. Neither of the main effects of time was significant on their own in post hoc testing. There were no differences found on SOL and SE. Overall, these results are equivocal; the significant finding in WASO is small and unlikely to be of practical significance. Moreover, this interaction was significant in the ITT analysis, but was not significant in the completer analysis (see Supplemental Material). As with our PSQI findings, they suggest that a more intensive dose and duration of mindfulness training are likely to be needed before robust objective changes can be observed.

\section{Limitations and Future Research}

The current experiment shows that a 4-week introductory mindfulness course has moderate effects on reducing cognitive arousal prior to sleep, and that these effects are correlated with improvements in subjective sleep quality. Our data support the theory that mindfulness practice enhances metacognitive control, which in turn reduces anxiety, rumination, and distraction in the pre-sleep period (Ong et al. 2012). Some strengths of the study are that participant dropout rates were low, and course attendance was good, indicating that we were adequately powered to detect treatment effects. We were also able to assess both subjective and objective sleep quality with a rigorous protocol for collecting and scoring the actigraphic data.

The study also has several limitations that might threaten its validity. As this was a sample of convenience, we were ultimately only able to carry out random assignment for 39 out of our 96 participants. As such, we cannot rule out the possibility of biased assignment in the last 3 blocks of the study. In particular, it is conceivable that participants who self-selected into the earlier class (and who subsequently formed the treatment group) may have been experiencing higher levels of stress, or had other reasons for wanting to get mindfulness training more urgently. Nevertheless, baseline characteristics were largely similar between the two groups, and when sleep variables did differ, this was in favor of the treatment group (more TIB and TST). We were also unable to collect follow-up data to assess whether the improvements in pre-sleep cognitive arousal were maintained over time. Finally, as our population was predominantly Singapore-born Han Chinese, the generalizability of our findings to other ethnicities and populations needs to be addressed in future studies.
Acknowledgments We acknowledge the assistance of Nicholas Chee, James Teng, Jia Lin, and Kian Foong Wong in data collection and scoring the actigraphy data, and Ju Lynn Ong for feedback and discussion.

Author Contributions ZH: executed the study, assisted with the data analysis, and collaborated in writing the paper. EL: assisted in conducting the intervention and wrote part of the methods. JCL: assisted with data analysis and the editing of the final manuscript. JL: designed the study, analyzed the data, and wrote the paper.

Funding Source This study was funded from a STaR investigator grant (NMRC/STaR/0015/2013) to Michael Chee (PI), and the National Research Foundation (Singapore) Science of Learning Grant (NRF2016-SOL002-001) to Julian Lim, June Lo, and Michael Chee (PI).

\section{Compliance with Ethical Standards}

Conflict of Interest The authors declare that they have no conflict of interest.

Ethical Approval This study was approved by the National University of Singapore Institutional Review Board, was conducted in accordance with the ethical standards of the 1964 Helsinki declaration and its later amendments.

Informed Consent Written informed consent was obtained from all participants prior to participation. Volunteers below 18 years of age additionally obtained parental consent to participate in this research.

Open Access This article is distributed under the terms of the Creative Commons Attribution 4.0 International License (http:// creativecommons.org/licenses/by/4.0/), which permits unrestricted use, distribution, and reproduction in any medium, provided you give appropriate credit to the original author(s) and the source, provide a link to the Creative Commons license, and indicate if changes were made.

\section{References}

Baer, R. A., Smith, G. T., Lykins, E., Button, D., Krietemeyer, J., Sauer, S., ... \& Williams, J. M. G. (2008). Construct validity of the Five Facet Mindfulness Questionnaire in meditating and snonmeditating samples. Assessment, 15(3), 329-342.

Black, D. S., O’Reilly, G. A., Olmstead, R., Breen, E. C., \& Irwin, M. R. (2015). Mindfulness meditation and improvement in sleep quality and daytime impairment among older adults with sleep disturbances: a randomized clinical trial. JAMA Internal Medicine, 175(4), 494-501.

Blake, M., Schwartz, O., Waloszek, J. M., Raniti, M., Simmons, J. G., Murray, G., ... \& Dudgeon, P. (2017). The SENSE study: treatment mechanisms of a cognitive behavioral and mindfulness-based group sleep improvement intervention for at-risk adolescents. Sleep, 40(6).

Bonnet, M. H., \& Arand, D. L. (1997). Hyperarousal and insomnia. Sleep Medicine Reviews, 1(2), 97-108.

Britton, W. B., Haynes, P. L., Fridel, K. W., \& Bootzin, R. R. (2010). Polysomnographic and subjective profiles of sleep continuity before and after mindfulness-based cognitive therapy in partially remitted depression. Psychosomatic Medicine, 72(6), 539-548.

Buysse, D. J., Reynolds, C. F., 3rd, Monk, T. H., Berman, S. R., \& Kupfer, D. J. (1989). The Pittsburgh Sleep Quality Index: a new instrument for psychiatric practice and research. Psychiatry Research, 28(2), 193-213.

Cappuccio, F. P., D’Elia, L., Strazzullo, P., \& Miller, M. A. (2010a). Quantity and quality of sleep and incidence of type 2 diabetes: a systematic review and meta-analysis. Diabetes Care, 33(2), 414-420. 
Cappuccio, F. P., D’Elia, L., Strazzullo, P., \& Miller, M. A. (2010b). Sleep duration and all-cause mortality: a systematic review and metaanalysis of prospective studies. Sleep, 33(5), 585-592.

Cappuccio, F. P., Cooper, D., D’Elia, L., Strazzullo, P., \& Miller, M. A. (2011). Sleep duration predicts cardiovascular outcomes: a systematic review and meta-analysis of prospective studies. European Heart Journal, 32(12), 1484-1492.

Chang, V. Y., Palesh, O., Caldwell, R., Glasgow, N., Abramson, M., Luskin, F., ... \& Koopman, C. (2004). The effects of a mindfulness-based stress reduction program on stress, mindfulness self-efficacy, and positive states of mind. Stress and Health: Journal of the International Society for the Investigation of Stress, 20(3), 141-147.

Chen, H. C., Lin, C. M., Lee, M. B., \& Chou, P. (2011). The relationship between pre-sleep arousal and spontaneous arousals from sleep in subjects referred for diagnostic polysomnograms. Journal of the Chinese Medical Association, 74(2), 81-86.

Dinges, D. F., Pack, F., Williams, K., Gillen, K. A., Powell, J. W., Ott, G. E., et al. (1997). Cumulative sleepiness, mood disturbance, and psychomotor vigilance performance decrements during a week of sleep restricted to 4-5 hours per night. Sleep, 20(4), 267-277.

Efird, J. (2011). Blocked randomization with randomly selected block sizes. International Journal of Environmental Research and Public Health, 8(1), 15-20.

Grandner, M. A., Kripke, D. F., Yoon, I. Y., \& Youngstedt, S. D. (2006). Criterion validity of the Pittsburgh Sleep Quality Index: investigation in a non-clinical sample. Sleep and Biological Rhythms, 4(2), 129-139.

Greeson, J. M., Zarrin, H., Smoski, M. J., Brantley, J. G., Lynch, T. R., Webber, D. M., ... \& Wolever, R. Q. (2018). Mindfulness meditation targets transdiagnostic symptoms implicated in stress-related disorders: understanding relationships between changes in mindfulness, sleep quality, and physical symptoms. Evidence-Based Complementary and Alternative Medicine, 2018, 4505191.

Gross, C. R., Kreitzer, M. J., Reilly-Spong, M., Wall, M., Winbush, N. Y., Patterson, R., ... \& Cramer-Bornemann, M. (2011). Mindfulnessbased stress reduction versus pharmacotherapy for chronic primary insomnia: a randomized controlled clinical trial. Explore: The Journal of Science and Healing (NY), 7(2), 76-87.

Haack, M., \& Mullington, J. M. (2005). Sustained sleep restriction reduces emotional and physical well-being. Pain, 119(1-3), 56-64.

Harvey, A. G. (2002). A cognitive model of insomnia. Behaviour Research and Therapy, 40(8), 869-893.

Horne, J. A., \& Ostberg, O. (1976). A self-assessment questionnaire to determine morningness-eveningness in human circadian rhythms. International Journal of Chronobiology, 4(2), 97-110.

Howell, A. J., Digdon, N. L., Buro, K., \& Sheptycki, A. R. (2008). Relations among mindfulness, well-being, and sleep. Personality and Individual Differences, 45, 773-777.

Jankowski, T., \& Holas, P. (2014). Metacognitive model of mindfulness. Consciousness and Cognition, 28, 64-80.

Kazdin, A. E. (1974). Reactive self-monitoring: the effects of response desirability, goal setting, and feedback. Journal of Consulting and Clinical Psychology, 42(5), 704-716.

Kok, B. E., \& Singer, T. (2017). Phenomenological fingerprints of four meditations: differential state changes in affect, mind-wandering, meta-cognition, and interoception before and after daily practice across 9 months of training. Mindfulness, 8(1), 218-231.

Landry, G. J., Best, J. R., \& Liu-Ambrose, T. (2015). Measuring sleep quality in older adults: a comparison using subjective and objective methods. Frontiers in Aging Neuroscience, 7, 166.

Lengacher, C. A., Reich, R. R., Paterson, C. L., Jim, H. S., Ramesar, S., Alinat, C. B., et al. (2015). The effects of mindfulness-based stress reduction on objective and subjective sleep parameters in women with breast cancer: a randomized controlled trial. Psycho-Oncology, 24(4), $424-432$.
Lim, J., \& Dinges, D. F. (2010). A meta-analysis of the impact of shortterm sleep deprivation on cognitive variables. Psychological Bulletin, 136(3), 375-389.

Lowe, C. J., Safati, A., \& Hall, P. A. (2017). The neurocognitive consequences of sleep restriction: a meta-analytic review. Neuroscience \& Biobehavioral Reviews, 80, 586-604.

Morin, C. M. (1993). Insomnia: psychological assessment and management. New York: Guilford Press.

Nicassio, P. M., Mendlowitz, D. R., Fussell, J. J., \& Petras, L. (1985). The phenomenology of the pre-sleep state: the development of the pre-sleep arousal scale. Behaviour Research and Therapy, 23(3), 263-271.

Ong, J. C., Ulmer, C. S., \& Manber, R. (2012). Improving sleep with mindfulness and acceptance: a metacognitive model of insomnia. Behavior Research and Therapy, 50(11), 651-660.

Ong, J. C., Manber, R., Segal, Z., Xia, Y., Shapiro, S., \& Wyatt, J. K. (2014). A randomized controlled trial of mindfulness meditation for chronic insomnia. Sleep, 37(9), 1553-1563.

Schutte-Rodin, S., Broch, L., Buysse, D., Dorsey, C., \& Sateia, M. (2008). Clinical guideline for the evaluation and management of chronic insomnia in adults. Journal of Clinical Sleep Medicine, 4(5), 487-504.

Sibinga, E. M., Perry-Parrish, C., Chung, S. E., Johnson, S. B., Smith, M., \& Ellen, J. M. (2013). School-based mindfulness instruction for urban male youth: a small randomized controlled trial. Preventive Medicine, 57(6), 799-801.

Strine, T. W., \& Chapman, D. P. (2005). Associations of frequent sleep insufficiency with health-related quality of life and health behaviors. Sleep Medicine, 6(1), 23-27.

Tan, N. C., Tan, M. S., Hwang, S. W., Teo, C. C., Lee, Z. K. N., Soh, J. Y. J., et al. (2016). Sleep time and pattern of adult individuals in primary care in an Asian urbanized community: a cross-sectional study. Medicine, 95(35), e4749.

Tan, A., Cisulli, P. A., Hong, Y., Tan, L. W. L., Van Dam, R. M., \& Bin, Y. S. (2017). Self-reported sleep characteristics in a multi-ethnic asian population: the Singapore health studies. Sleep Medicine, 40(S1), e35.

Tang, N. K., \& Harvey, A. G. (2004). Effects of cognitive arousal and physiological arousal on sleep perception. Sleep, 27(1), 69-78.

Teasdale, J. D. (1999). Metacognition, mindfulness and the modification of mood disorders. Clinical Psychology and Psychotherapy, 6, 146-155.

Tennant, R., Hiller, L., Fishwick, R., Platt, S., Joseph, S., Weich, S., et al. (2007). The Warwick-Edinburgh mental well-being scale (WEMWBS): development and UK validation. Health and Quality of Life Outcomes, 5, 63.

Todd, J., \& Mullan, B. (2014). The role of self-monitoring and response inhibition in improving sleep behaviours. International Journal of Behavioral Medicine, 21(3), 470-477.

Walch, O. J., Cochran, A., \& Forger, D. B. (2016). A global quantification of "normal" sleep schedules using smartphone data. Science Advances, 2(5), e1501705.

Wild, C. J., Nichols, E. S., Battista, M. E., Stojanoski, B., \& Owen, A. M. (2018). Dissociable effects of self-reported daily sleep duration on high-level cognitive abilities. Sleep, 41(12), zsy182.

Williams, K. A., Kolar, M. M., Reger, B. E., \& Pearson, J. C. (2001). Evaluation of a wellness-based mindfulness stress reduction intervention: a controlled trial. American Journal of Health Promotion, 15(6), 422-432.

Yong, L. C., Li, J., \& Calvert, G. M. (2017). Sleep-related problems in the US working population: prevalence and association with shiftwork status. Occupational and Environmental Medicine, 74(2), 93-104.

Zhang, J. X., Liu, X. H., Xie, X. H., Zhao, D., Shan, M. S., Zhang, X. L., ... \& Cui, H. (2015). Mindfulness-based stress reduction for chronic insomnia in adults older than 75 years: a randomized, controlled, single-blind clinical trial. Explore, 11(3), 180-185.

Publisher's Note Springer Nature remains neutral with regard to jurisdictional claims in published maps and institutional affiliations. 